\title{
A Low Cost and Low Power Consumption Automatic Water Meter Reading System: Hardware Investigation and Network Design
}

\author{
Lap-Luat Nguyen, Huu-Tue Huynh, Tuan-Duc Nguyen \\ School of Electrical Engineering, International University, Ho Chi Minh City, Vietnam \\ Correspondence: Tuan-Duc Nguyen, ntduc@hcmiu.edu.vn \\ Manuscript communication: received 20 December 2014, accepted 4 May 2015
}

\begin{abstract}
This paper presents a low power consumption and low cost automatic data collection network for water meter application. Based on transmission performance and power consumption, several low cost sub-GHz wireless transceivers are analyzed and compared, and consequently a suitable hardware is chosen. The associated network protocol stack is also examined. To construct the automatic collecting data mechanism, we consider a cluster based wireless sensor network (WSN) where routers and a GPRS gateway are used to link each cluster to a data collection center. Advantages of this proposed configuration are the simple implementation, low cost and low power consumption. By using the Monte Carlo simulation technique, packet delivery ratio and power consumption for different topologies are investigated. Based on obtained results, the optimum network topology for automatic water meter reading in a typical urban environment is finally proposed.
\end{abstract}

Keywords- Wireless sensor network, low cost and low power consumption network, automatic water meter reading.

This research was supported by the Vietnam's National University of Hochiminh-city project: “Energy consumption optimization of cooperative communication techniques in cognitive radio network and wireless sensor network".

\section{INTRODUCTION}

Due to obsolete infrastructures and with rapidly growing population and households, present data collection methods occasionally lead to system unreliability or data loss; the water supplying problem has become more difficult to solve in rapidly growing countries like Vietnam [1]. In this context, automatic and continuous reliable water metering is an important requirement.

There are several solutions for automatic water meter reading. First, transmitting water data using Mbus [2] or power line link structure [3-5]; but it has some disadvantages such as system vulnerability and heavy workload of wiring. It is therefore difficult to be deployed in urban situation of developing countries. Wireless technology using radio frequency communication such as fixed base station [6] or mobile radio station methods $[7,8]$ recently becomes popular, due to the fact that it is easier to deploy and at a cheaper price than cable. Hence, building a wireless collecting data system for water metering application is more practical than building a wired system. Many research studies on water monitoring based on GPRS have been proposed in the last few years [9]. However, the price of GPRS modems and GPRS services is quite expensive; henceforth, the application of GPRS technology to every household is not practical $[10,11]$.

In recent years, the wireless sensor network (WSN) technology has been applied to automatic meter reading domain for many reasons. Besides its low cost and low energy consumption, WSNs can be easily deployed with the coverage area easily expanded. It has been implemented for automatic water meter reading in a whole city as an experimental system [12]. With low power consumption, WSNs can last up to more than 5 years on normal battery [13]. ZigBee, a wireless networking standard that is aimed for remote control and sensor applications (working at $2.4 \mathrm{GHz}$ frequency band), is usually chosen for some automatic wireless applications $[13,14]$.

In practice, besides its advantages previously mentioned, there is a concern about the standard frequency which is reserved for wireless metering application. Many important semiconductor companies have introduced to the consumer market some new transceivers to be used in automatic meter reading application, working at the frequency band $433-868 \mathrm{MHz}$ instead of $2.4 \mathrm{GHz}[15,16]$. These transceivers with this frequency band standard will be developed and maintained everywhere around the world.

This paper proposes a new design of an automatic water meter reading network based on the WSN technology. By using simulation tools, an optimized topology with appropriate hardware is suggested. In order to confirm the validity of this new design, we compare the proposed network performance, including the power consumption, with some other known solutions. This comparison shows that our proposed solution is effective. In fact, by constructing a network with low cost, low power consumption, with good performance using the world's common standard would offer people an opportunity to apply it in practice.

This paper is organized as follows. Section 2 discusses elements that influence the design and then 


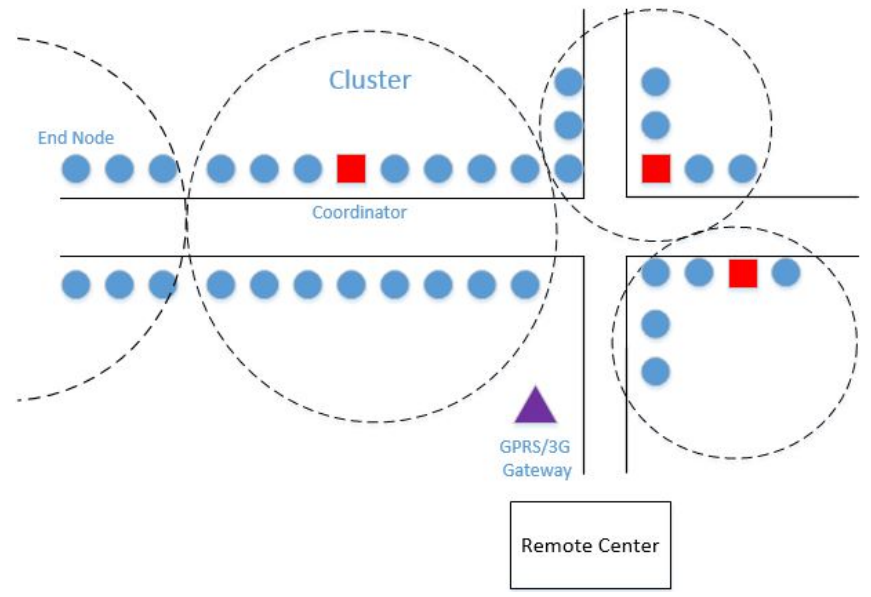

Figure 1. Simple network topology design for distribution metering.

comes up with the proposed topology. In Section 3, water metering circuits are designed and a suitable wireless transceiver is investigated. In order to find out the optimized topology, network performance and network energy efficiency based on simulation are presented in Section 4. The conclusion is finally given in Section 5 .

\section{Network Design for Automatic Meter READING}

Model of one residential area of an urban city in developing countries that has a crossroad with many houses along two sides of streets is illustrated in Figure 1. With the goal of building an automatic and ubiquitous water metering collection data network, the automatic data collection network topology is proposed based on clusters, as also shown in Figure 1.

Consider a situation where in a water meter hardware of every household is integrated with a wireless transceiver node (End Node in Figure 1). The water meter network is divided into several clusters for a simple management and a practical deployment. Each cluster has one Coordinator node that manages many End Nodes. The role of every End Node is to get water metering data from the water meter, then to transmit these data to the coordinating node. After receiving data from end nodes, the coordinating node will transfer the data to the GPRS/3G Gateway (for inter-networking by using GPRS/3G cellular network) in order to forward these data to the Remote center.

\section{Water Meter and Automatic Data Collection Network}

\subsection{Water Metering Circuit}

The water metering circuit is designed to record the amount of used water at every household, by converting the water metering data from analog to digital data. All of current water meters consist of a wheel where one or more magnets are attached to that wheel. When

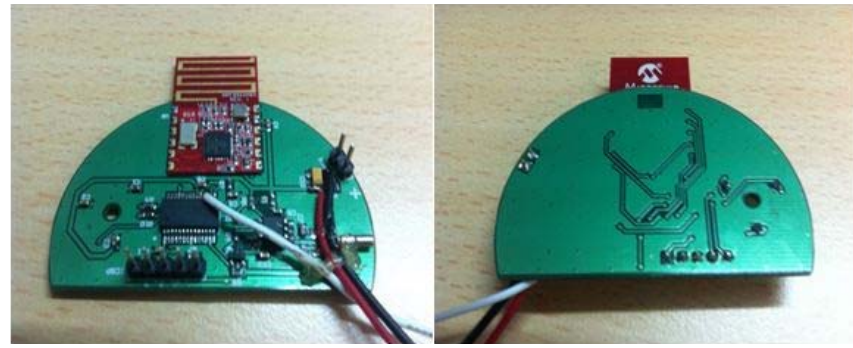

Figure 2. Water metering designed circuit.

Table I

HARDWARE COMPARISON OF WSN Nodes

\begin{tabular}{|c|c|c|c|}
\hline & $\begin{array}{l}\text { MC13224V } \\
\text { (Microchip ZigBee) }\end{array}$ & $\begin{array}{l}\text { CC1120 } \\
\text { (Texas Instrument) }\end{array}$ & $\begin{array}{l}\text { CC1110 } \\
\text { (Texas Instrument) }\end{array}$ \\
\hline Standard & IEEE 802.15 .4 & $\begin{array}{l}\text { IEEE } 802.15 .4 \mathrm{~g} \\
\text { systems Ghz }\end{array}$ & $\begin{array}{l}\text { TI Proprietary } \\
868 / 915 / 433 \mathrm{MHz}\end{array}$ \\
\hline Data rate & $250 \mathrm{Kbps}$ & 0 to $200 \mathrm{kbps}$ & up to $500 \mathrm{kbps}$ \\
\hline Output power & $\begin{array}{l}-30 \mathrm{dBm} \\
\text { to }+4 \mathrm{dBm}\end{array}$ & $\begin{array}{l}-40 \mathrm{dBm} \\
\text { to } 16 \mathrm{dBm}\end{array}$ & $\begin{array}{l}-6 \mathrm{dBm} \\
\text { to } 10 \mathrm{dBm}\end{array}$ \\
\hline $\begin{array}{l}\text { Received } \\
\text { Sensitivity }\end{array}$ & $\begin{array}{l}-96 \mathrm{dBm} \\
\text { for } \mathrm{DCD} \text { mode } \\
-100 \mathrm{dBm} \\
\text { for NDC mode }\end{array}$ & $\begin{array}{l}-123 \mathrm{dBm} \\
\text { for } 1.2 \mathrm{kbps} \\
-110 \mathrm{dBm} \\
\text { for } 50 \mathrm{kbps}\end{array}$ & $\begin{array}{l}-110 \mathrm{dBm} \\
\text { for } 1.2 \mathrm{kbps}\end{array}$ \\
\hline $\begin{array}{l}\text { Frequency } \\
\text { band }\end{array}$ & $\begin{array}{l}2.4 \mathrm{GHz} \text { ISM } \\
\text { Band }\end{array}$ & $\begin{array}{l}170 / 315 / 433 \\
868 / 915 / 950 \\
\text { MHz ISM/SRD }\end{array}$ & $\begin{array}{l}315 / 433 / 868 \\
\text { and } 915 \mathrm{MHz} \\
\text { ISM/SRD }\end{array}$ \\
\hline $\begin{array}{l}\text { Indoor } \\
\text { transmission } \\
\text { test }\end{array}$ & $\begin{array}{l}\text { Can go through } \\
2 \text { walls }\end{array}$ & $\begin{array}{l}\text { Long range for } \\
\text { maximum indoor } \\
\text { penetration }\end{array}$ & $\begin{array}{l}\text { Can go } \\
\text { through } \\
7 \text { walls }\end{array}$ \\
\hline $\begin{array}{l}\text { Outdoor } \\
\text { transmission } \\
\text { test }\end{array}$ & $200 \mathrm{~m}$ & $\begin{array}{l}\text { Beyond } \\
10 \mathrm{Km}\end{array}$ & $\begin{array}{l}550 \mathrm{~m} \text { with some } \\
\text { obstacles } \\
\mathrm{Up} \text { to } 1 \mathrm{Km} \text { in } \\
\text { free space }\end{array}$ \\
\hline $\begin{array}{l}\text { Power } \\
\text { Consumption }\end{array}$ & $\begin{array}{l}\text { RX: } 22 \mathrm{~mA} \\
\text { TX: } 29 \mathrm{~mA} \\
\text { Sleep: } 0.85 \mu \mathrm{A}\end{array}$ & $\begin{array}{l}\text { RX: } 17 \mathrm{~mA} \\
\text { TX: } 45 \mathrm{~mA} \\
\text { Sleep: } 0.3 \mu \mathrm{A}\end{array}$ & $\begin{array}{l}\text { RX: } 18.7 \mathrm{~mA} \\
\text { TX: } 21 \mathrm{~mA} \\
\text { Sleep: } 0.5 \mu \mathrm{A}\end{array}$ \\
\hline $\mathrm{MCU}$ & 32 bit ARM7 & No MCU & $\begin{array}{l}\text { Integrated } 8051 \\
\text { MCU }\end{array}$ \\
\hline
\end{tabular}

an amount of water goes through a water meter, this wheel will spin in order to show how many litres of water were used by this household. This water metering circuit will be integrated with a current water meter for record used water data by calculating the number of wheel rotations.

A designed circuit (as shown in Figure 2) includes three hall sensors to detect the spins of the magnetic wheel, an MCU (PIC18F26K22) to process data and a low cost, low power consumption transceiver module (which is described in the next section). The schematic diagram of this designed circuit is illustrated in Figure 3.

\subsection{Wireless Hardware Selection}

This section focuses on the study of an appropriate wireless sensor network hardware which satisfies the following goals: low cost, low power consumption, reliable transmission in urban environments. With a comparison of some latest transceiver hardware on the market in Table I, a suitable transceiver will be chosen based on these criteria.

According to Table I, Texas Instrument CC1110 and Microchip MC133224V have the advantage of an inte- 

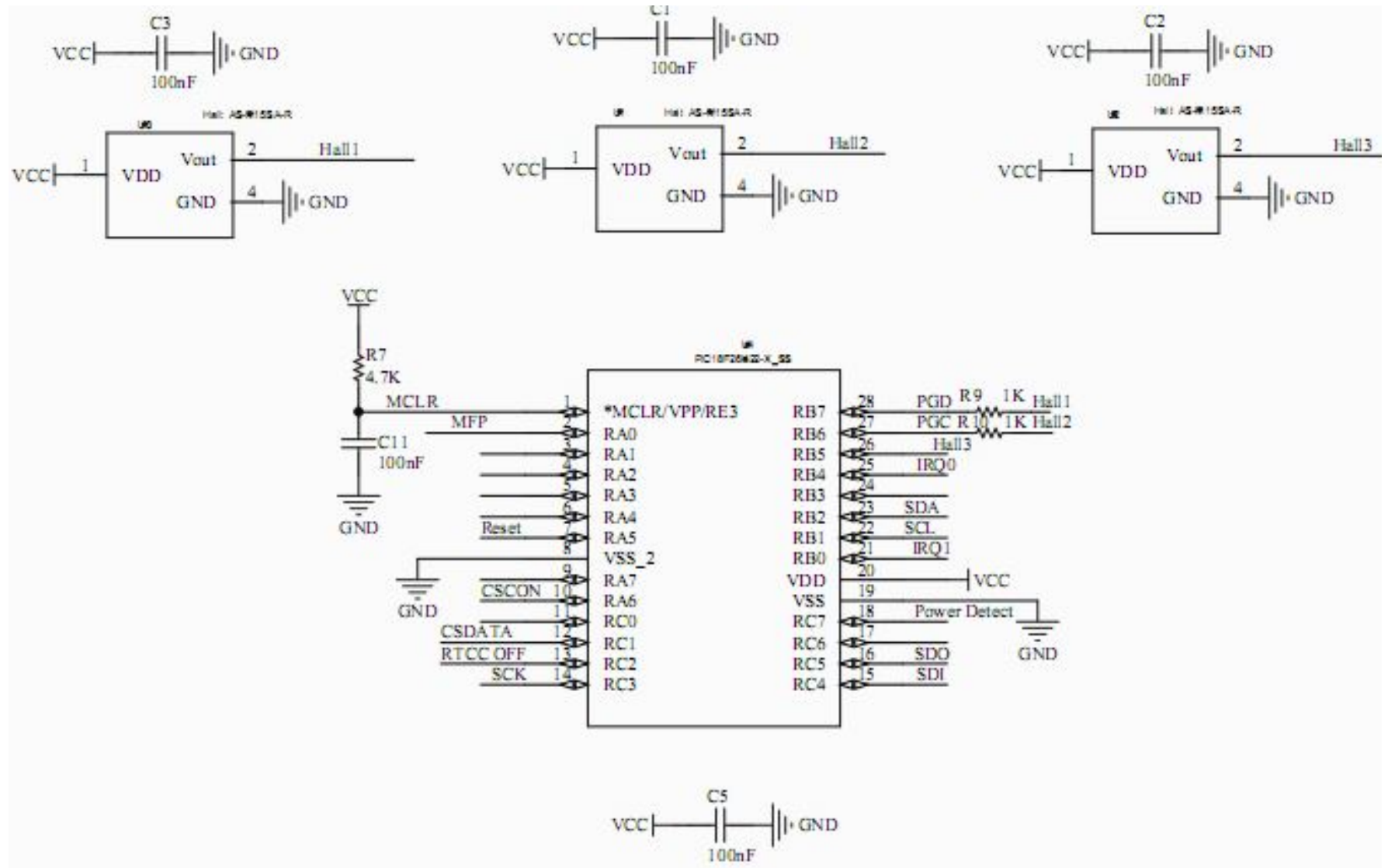

Figure 3. Water metering designed circuit schematic.
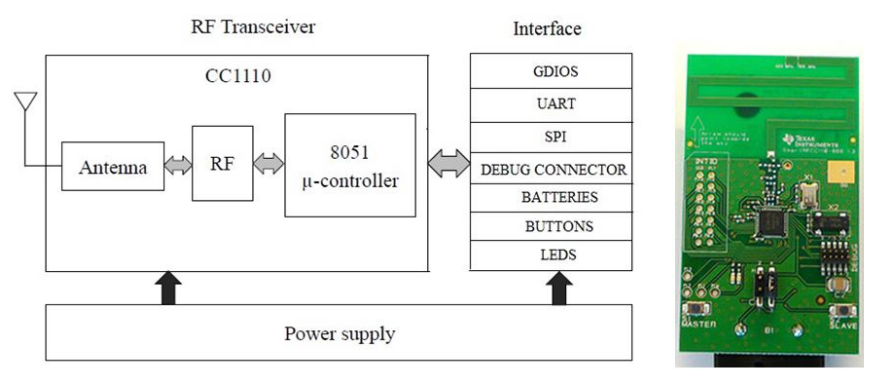

Figure 4. CC1110 mini development kit and its block diagram.

grated MCU (Micro-controller unit), yielding a lower cost of a wireless module. The power consumption of CC1110 and MC13224V SoCs (including the MCU) are equal, but CC1110 has the advantage of a reliable transmission distance due to the lower received sensitivity and the higher transmission out-put power. In case of transmission range, CC1120 has the longest transmission (up to $10 \mathrm{Km}$ in datasheet), but with higher cost and power consumption than CC1110.

In Figure 5, we perform a practical range testing of the CC1110 module in a real case of semi-urban, city and indoor environments. Results of transmission distance test in Table II prove that the CC1110 transmission range is very appropriate and suitable for the proposed Automatic Water Meter network where the node-tocoordinator distance is typically less than 500 meters.

Another advantage is the SoC design of CC1110, which allows a small size wireless module to be in-
Table II

Testing Range Result Table.

\begin{tabular}{|l|l|l|}
\hline & $0 \mathrm{dBm}$ & $10 \mathrm{dBm}$ \\
\hline Line of sight & $1.2 \mathrm{Km}$ & $1.5 \mathrm{~km}$ \\
\hline Obstacle & $430 \mathrm{~m}$ & $550 \mathrm{~m}$ \\
\hline Indoor testing & 5 walls & 7 walls \\
\hline
\end{tabular}

tegrated in the water meter circuit. Moreover, with the support of Texas Instrument network protocols (SimpliciTi and wireless M-Bus) for CC1110 transceiver, we can design an efficient star routing network for one Cluster in Figure 1, with a support of range extender in order to extend the network coverage.

\section{Simulation and Results}

In this section, by using the network simulation 2 (NS-2) tool, the proposed network is simulated with many scenarios (by changing network and hardware parameters) in order to evaluate the packet collision ratio, transmission latency and energy consumption. The goal of this simulation is to determine a practical number of nodes per one cluster, node-to-node distance and transmission output power.

We used a NS-2 library code of IEEE 802.15.4 standard (defined by The City College of New York and Samsung Advanced Institute of Technology, USA [17]) 


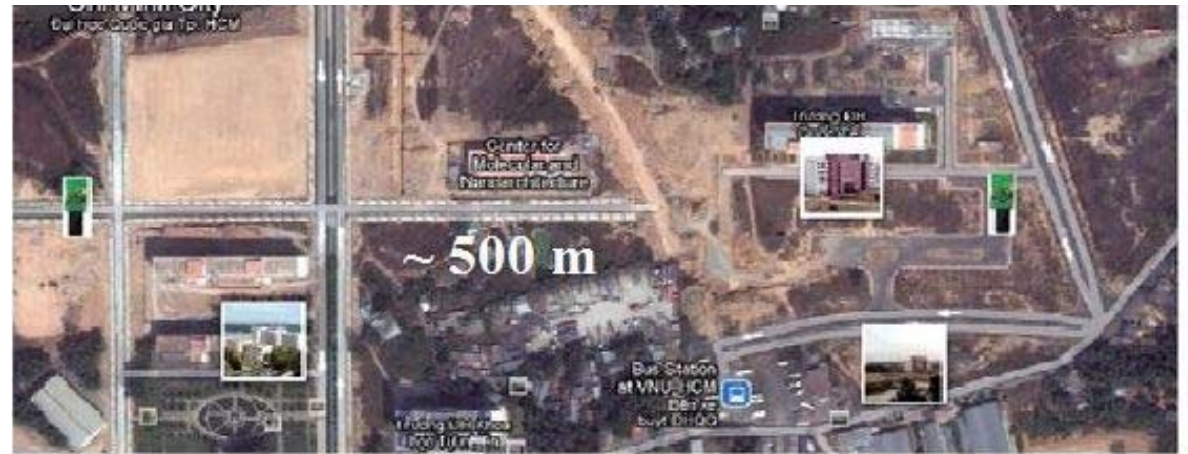

(a)

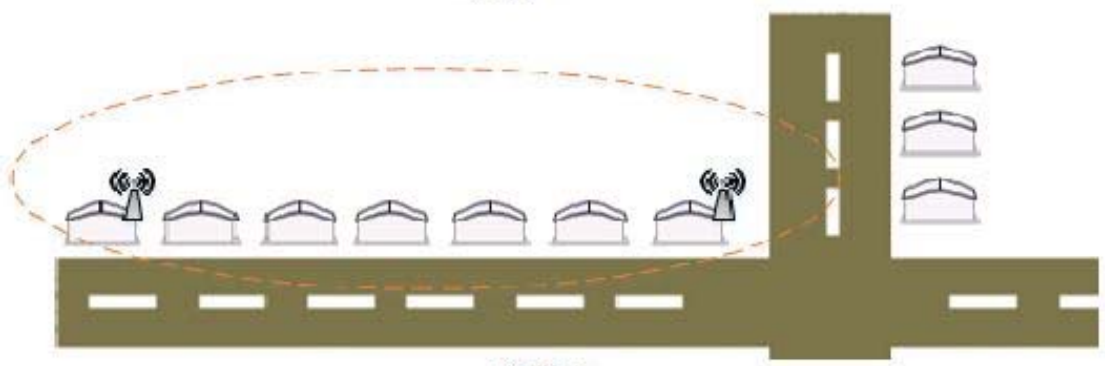

(b)

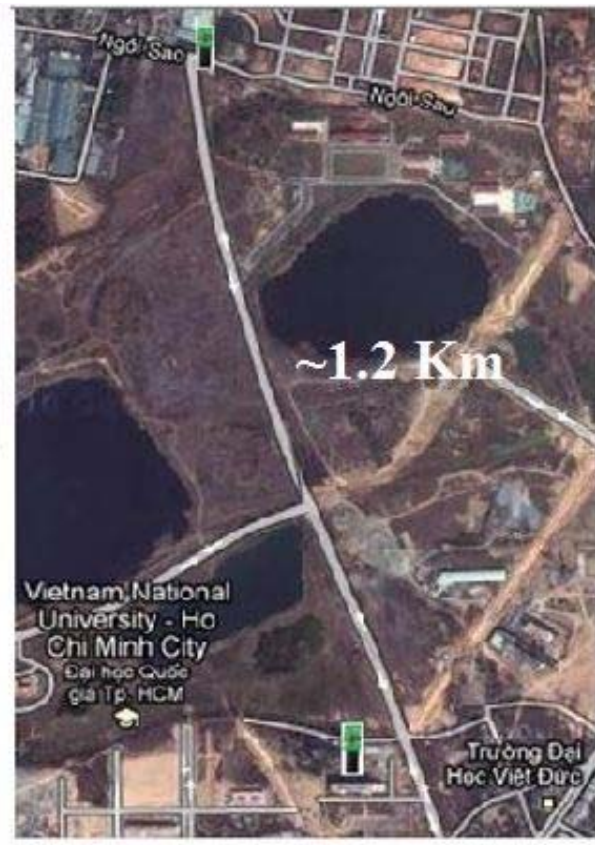

(c)

Figure 5. CC1110 real case range testing. (a) Testing through obstacles (b) Urban environment testing (c) Free-space line-of-sight.

Table III

Simulation PARAmeters.

\begin{tabular}{|l|l|}
\hline Protocol & SimpliciTI \\
\hline Antenna & Ommi Antenna \\
\hline Propagation model & Two Ray Ground \\
\hline Transmitted power & $0 \mathrm{dBm}$ to $10 \mathrm{dBm}$ \\
\hline Received sensitivity & $-110 \mathrm{dBm}$ at $1.2 \mathrm{kbps}$ \\
\hline Distance node to node & 5 meters to 15 meters \\
\hline Number of nodes & 5 to 50 nodes \\
\hline Frequency band & $868 \mathrm{MHz}$ \\
\hline
\end{tabular}

and modified it to adapt to the SimpliciTI protocol. In physical layer, propagation model is chosen as Two Ray model. The number of nodes varies from 5 to 50 nodes for one cluster. The output power is set between $0 \mathrm{dBm}$ to $10 \mathrm{dBm}$. Node to node distance changes from 5 to 15 meters. In Table III, simulation parameters of the physical layer are shown. Based on the network topology design in Section 2, Figure 6 shows the network topology that is used in simulation scenarios.

\subsection{Packet Collision Ratio and Delay}

In Figure 7, simulations estimate the packet loss due to the packet collision when changing the size of the cluster (it means changing the number of nodes per cluster). The packets loss due to collision is calculated by

$$
\text { packet_loss }=\frac{\text { packet_sent }- \text { packet_receive }}{100} \text {. }
$$

Based on the simulation result in Figure 7, we can observe that number of lost packets due to collision

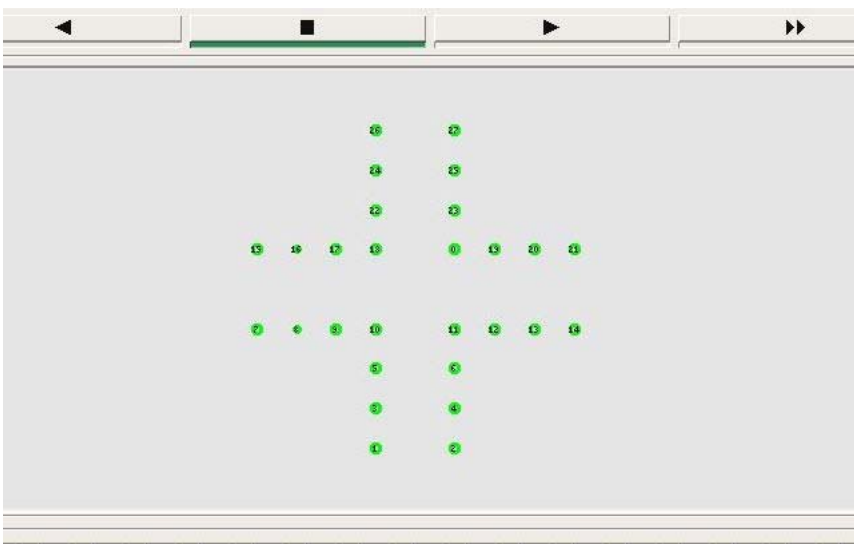

Figure 6. Network topology in simulation scenarios.

increases to $24 \%$ when the number of nodes in the network is 50. In a secured wireless transmission, CSMA/CA technique is used to resend a lost packet and to ensure all dropped packets will be resent correctly $100 \%$, so that the packet loss by collision less than $30 \%$ can be acceptable for a wireless network design.

In considering an actual urban population density in Hochiminh-city, as well as required facilities for deployment and maintenance, each cluster in our network should have 30 to 50 nodes.

In the next simulation scenario, the number of nodes per cluster is fixed at 30, but the transmitted power is changed from 0 to $10 \mathrm{dBm}$ and the node-to-node distance is also varied from 5 to 20 meters. The goal of this scenario is to observe the changing of packet loss by collision as function of the node-to-node distance.

When the node-to-node distance of cluster is less than 20 meters, $10 \mathrm{dBm}$ output power generates more 


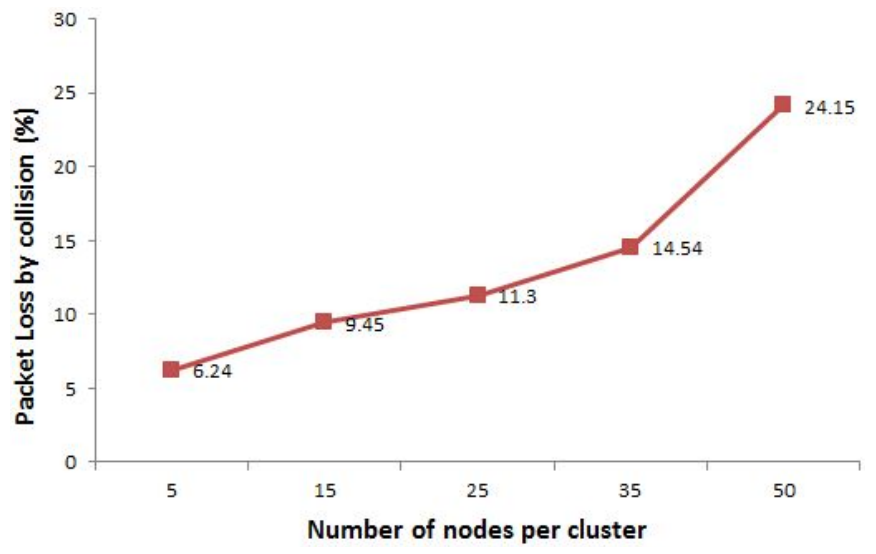

Figure 7. Packets collision loss when increasing cluster size.

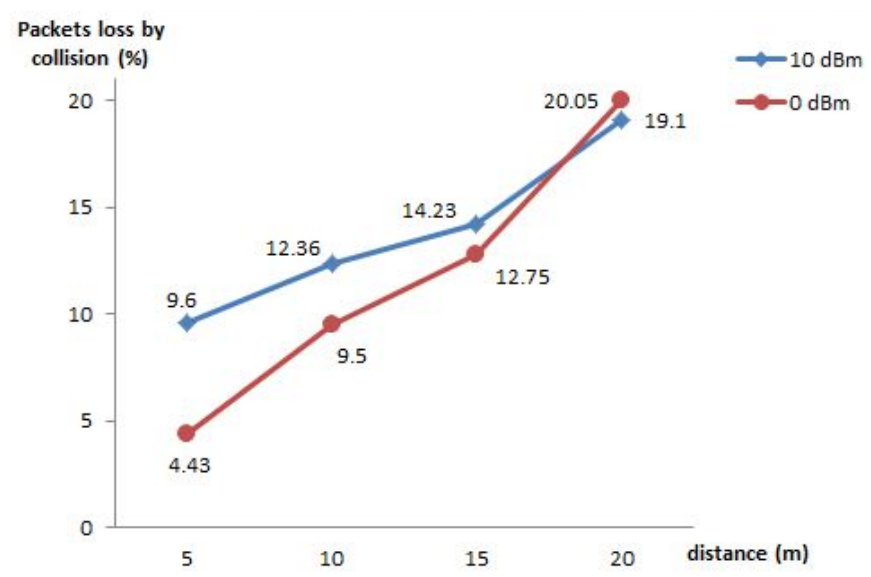

Figure 8. Packet loss with different output power and distances.

interference to other nodes than $0 \mathrm{dBm}$ output power. This is why the packet loss due to collision is higher. In the practical case of urban city where the distance of each household is less than 15 meters (i.e. the node-tonode distance between two nodes is less than 15 meters), based on Figure 8, the automatic data collection network can operate stably with packet loss by collision less than $12.75 \%$. Hence, the output power should be chosen at $0 \mathrm{dBm}$ instead of $10 \mathrm{dBm}$ in order to reduce the energy consumption of the wireless node.

The simulation result in Figure 9 illustrates the transmission delay time of a whole network when increasing the number of nodes. The packet size is set to 20 bytes and each node sends 400 packets. We can observe that the transmission delay time is less than $1000 \mathrm{~ms}$ when the number of nodes per cluster is up to 50 . We can have a conclusion that the transmission latency is less than 1 second with the number of nodes per cluster less than 50 .

\subsection{Energy Consumption}

Consider two AAA batteries to be used for one wireless end node and assume that one battery of $1.5 \mathrm{~V}$ delivers $900 \mathrm{mAh}$; the energy contained in the two batteries is $2700 \mathrm{mWh}$ (equal to 9720 Joules). We would like to warranty that batteries for a wireless end node should last at least 5 years; for this purpose we run

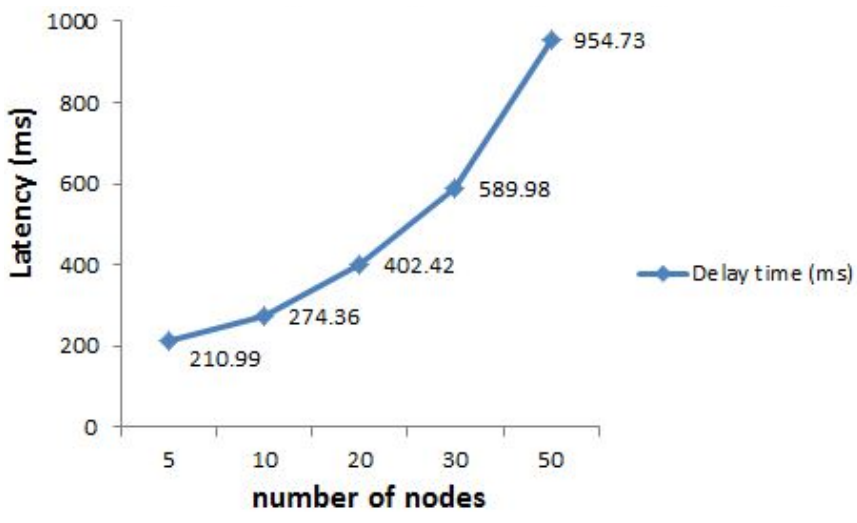

Figure 9. Latency of network.

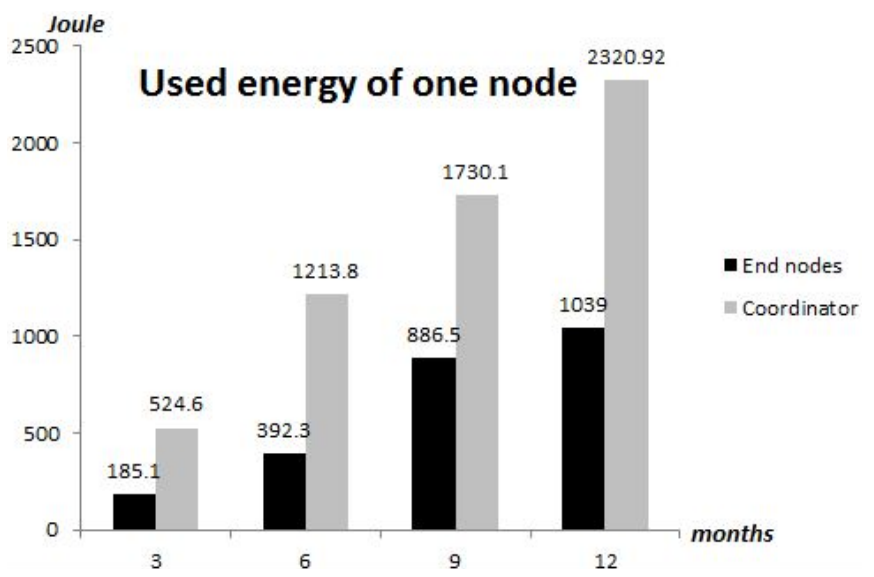

Figure 10. Energy consumption of one node after 1 year.

simulations for a cluster of 30 nodes in order to estimate the energy consumption of end and coordinator nodes for one year.

Let us assume that the remote center needs to collect meter data two times per day for tracking and maintaining the service, so that all end nodes have to transmit their data twice every day. To save energy, after finishing the transmission, all these End devices nodes should switch to the sleep mode.

Based on the transceiver datasheet [18], the current consumption of a wireless node (transceiver and MCU) active in the transmission mode is $21 \mathrm{~mA}$ (TX mode at $0 \mathrm{dBm}$ output power), in the reception mode is $18.7 \mathrm{~mA}$ ( $\mathrm{RX}$ mode), and in the sleep mode is 0.005 $\mathrm{mA}$. Figure 10 shows the energy consumption of the coordinator node and the average energy consumption of one End node in a cluster for 1 year.

We can observe that the energy consumption of the coordinator is always much higher than the consumption of one end node since it must receive packets all time and then forward these packets to the remote center while the end nodes are switching to the sleep mode. Moreover, the average energy consumption of the end node is 1039 Joules, so that the 9720 Joules energy contained in the two AAA batteries in one end node can last up to 9 years. 


\section{Conclusion}

In this paper, a design of a low cost and low power consumption wireless sensor network for an automatic water meter reading system in the urban environment is proposed. In the proposed network, meter hardware design, energy efficient wireless hardware and optimized data collection network topology have been investigated to ensure the automatic and long term operation with a normal AAA dual-battery power supply.

Through NS-2 network simulation, the packet loss due to collision, the packet delivery delay and the energy consumption of wireless nodes have also been performed in this paper.

\section{REFERENCES}

[1] N. T. N. Dung, "Management of urban water supplying in vietnam-situation and solution." [Online]. Available: http://http://www.xaydung.gov.vn/web/ guest/trang-chi-tiet/-/tin-chi-tiet/Z2jG/86/30459

[2] H. Ziegler and F. Physik, "The m-bus: A documentation rev.4.8." [Online]. Available: http://www.m-bus.com

[3] B. S. Park, D. H. Hyun, and S. K. Cho, "Implementation of AMR system using power line communication," EEE/PES Transmission and Distribution Conference and Exhibition: Asia Pacific, vol. 1, pp. 18-21, 2002.

[4] S. Y. Tan and M. Moghavvemi, "Pic-based automatic meter reading and control over the low voltage distribution network," SCOReD Student Conference on Research and Development Proceedings, Shah Alam, Malaysia, pp. 517520, 2002.

[5] S. K. Wong and M. Moghavvemi, "A robust system for data transmission over the row voltage distribution network," SCOReD Student Conference on Research and Development, pp. 399-402, 2002.

[6] B. S. Koay, "Design and implementation of bluetooth energy meter," Proceedings of the Joint Conference of the Fourth International Conference on Information, Communications and Signal Processing, and the Fourth Pacific Rim Conference on Multimedia, vol. 3, pp. 1474-1477, Dec. 2003.

[7] S. W. Lee, C. S. Wu, M. S. Chiou, and K. T. Wu, "Design of an automatic meter reading system," Proceedings of the IEEE 22nd International Conference on Industrial Electronics, Control, and Instrumentation, pp. 631-636, 1996.

[8] N. Q. Nhan, M. T. Vo, T. D. Nguyen, and H.-T. Huynh, "Improving the performance of mobile data collecting systems for electricity meter reading using wireless sensor network," International Conference on Advanced Technologies for Communications (ATC), pp. 241-246, 2012.

[9] Q. Wu, Y. Liang, Y. Sun, C. Zhang, and P. Liu, "Application of GPRS technology in water quality monitoring system," World Automation Congress (WAC), pp. 7-11, 2010.

[10] Z. Zi-han, Z. Xue-jiao, and Z. Wei, "Design and implementation of remote meter reading system," 2nd International Conference on Software Technology and Engineering (ICSTE), no. 2, pp. V2-247-V2-250, 2010.

[11] $\mathrm{W}$. $\mathrm{Wu}, \mathrm{W}$. $\mathrm{Wu}$, and $\mathrm{X}$. $\mathrm{Wu}$, "Research on long-range and metering reading for water meter based on GPRS," 2nd International Conference on Computer Engineering and Technology (ICCET), no. 7, pp. V7-725-V7-727, 2010.

[12] A. Zabasta and L. R. N Kunicina, Y Chaiko, "Automatic wireless meters reading for water distribution network in Talsi city," EUROCON - International Conference on Computer as a Tool (EUROCON), 2011 IEEE, pp. 1-4, 2011.

[13] Y.-W. Lee, S. Eun, and S.-H. Oh, "Wireless digital water meter with low power consumption for automatic me- ter reading," International Conference on Convergence and Hybrid Information Technology 2008, pp. 639-645, 2008.

[14] B. Zhang and J. Liu, "A kind of design schema of wireless smart water meter reading system based on ZigBee technology," International Conference on E-Product E-Service and E-Entertainment (ICEEE), pp. 1-4, 2010.

[15] Texas Instruments, "Texas instruments wireless product." [Online]. Available: http://focus.ti. com/wireless/docs/wirelessoverview.tsp?familyId= $2003 \&$ sectionId $=646 \&$ tabId $=2735$

[16] Microchip, "Microchip wireless product." [Online]. Available: http://www.microchip.com/stellent/idcplg? IdcService=SS_GET_PAGE\&nodeId=2664\&param = en535918

[17] T. C. C. of New York and S. A. I. of Technology, "Zigbee software modules." [Online]. Available: http: / / ees2cy.engr.ccny.cuny.edu/zheng/pub/

[18] Texas Instruments, CC1110 datasheet, Texas Instruments. [Online]. Available: www.ti.com/general/docs/lit

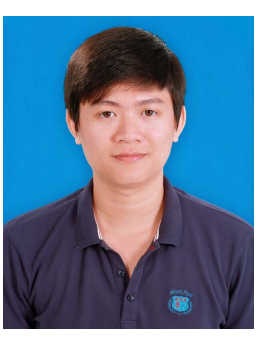

Lap-Luat Nguyen received the B.Eng. degree in electrical engineering from the International University of Vietnam National University, Ho Chi Minh City, in 2013. At that year, he received a full-scholarship and continued M.Eng at the International University. Since Sep. 2013, he has been accepted as Research Assistant at the School of Electrical Engineering, International University. His research interests include wireless sensor networks and communication networks.

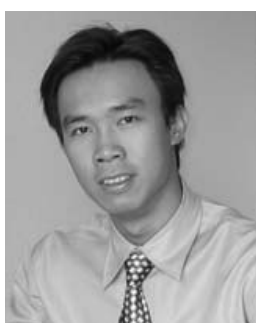

Tuan-Duc Nguyen received the M.Sc. degree in electrical engineering from Telecom ParisTech University, France, and the Ph.D. degree in signal processing for telecommunication from the University of Rennes 1, France, in 2005 and 2009, respectively. Since 2009, he has been a Lecturer and Researcher at the School of Electrical Engineering, International University of Vietnam National University, Ho Chi Minh City, Vietnam. His research interests include cooperative communications, wireless sensor networks, energy constrained wireless networks, and wireless ad hoc networks.

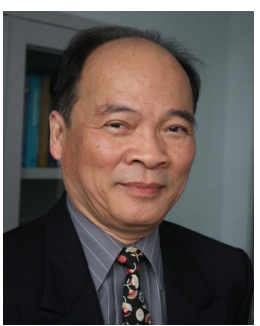

Huu-Tue Huynh was born in Hue, Vietnam. He received the Sc.D. degree in 1972 from Laval University, Canada, where from 1969 to 2004 he was professor of the Department of Electrical and Computer Engineering. In 2004, he left Laval University to become Chairman of the Department of Data Processing at The College of Technology of the Vietnam National University, Hanoi. During the period 2007-2011, he was the President of Bac Ha International University, Vietnam. He is now a research professor at the School of Electrical Engineering, International University, Vietnam National University, Ho Chi Minh City, Vietnam. He was an Invited Guest at The AT\&T Information Systems in Neptune, N.J. in 1984 and has been invited to give lectures at several Universities in Europe, America as well as in Asia. Professor Huynh is author and coauthor of two books and more than two hundred papers and research reports in Information Processing. He has served as Consultant to a number of Canadian Government Agencies and Industries. His research interests cover stochastic simulation techniques, information processing, fast algorithms and architecture with applications to finance and to communications. 